Document downloaded from:

http://hdl.handle.net/10251/102952

This paper must be cited as:

Pedrón-Torrecilla, J.; Rodrigo Bort, M.; M. Climent, A.; Liberos, A.; Pérez-David E; Bermejo, J.; Arenal, A.... (2016). Noninvasive Estimation of Epicardial Dominant High-Frequency Regions During Atrial Fibrillation. Journal of Cardiovascular Electrophysiology. 27(4):435442. doi:https://doi.org/10.1111/jce.12931

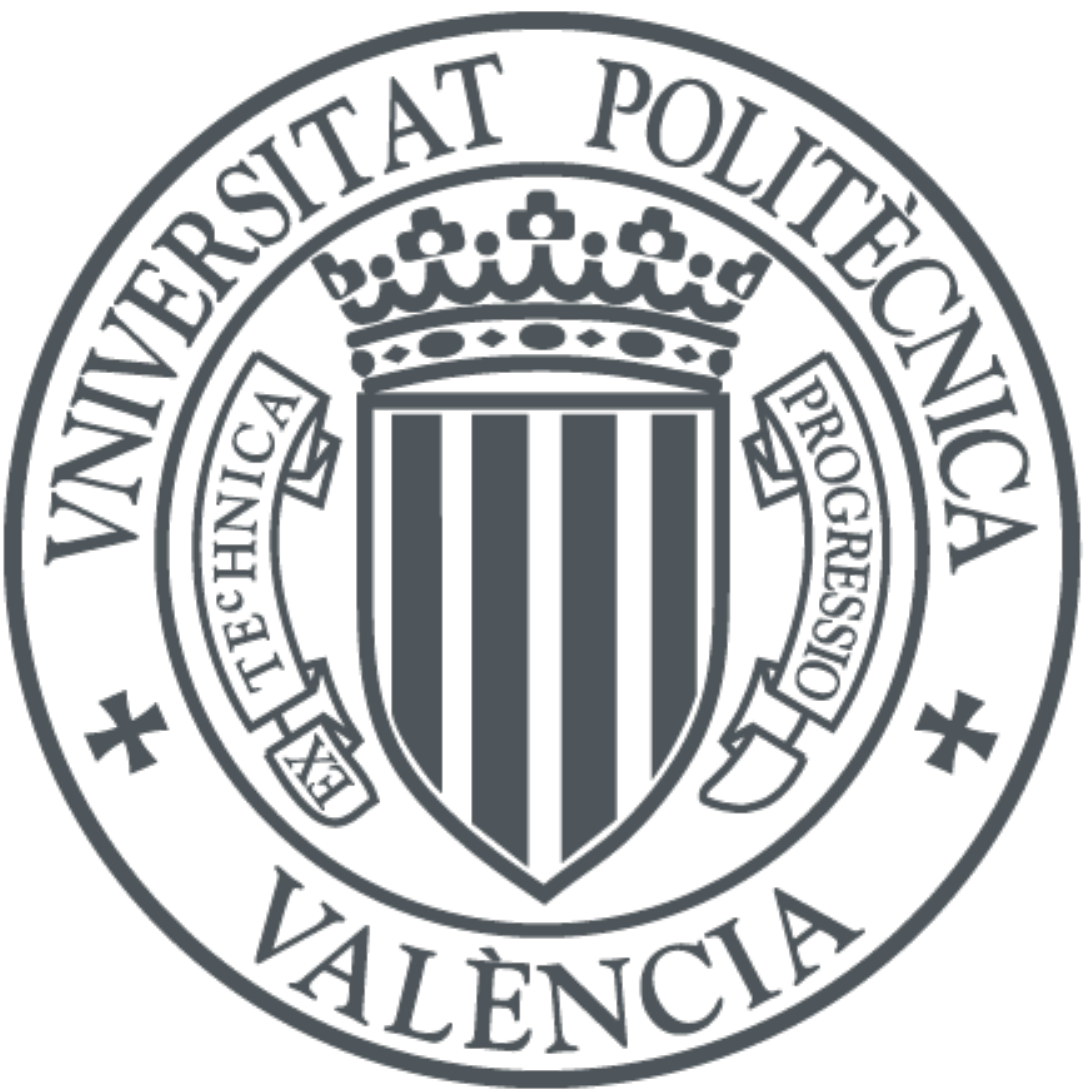

The final publication is available at

http://onlinelibrary.wiley.com/doi/10.1111/jce.12931/abstract

Copyright Blackwell Publishing

Additional Information 


\title{
Noninvasive Estimation of Epicardial Dominant High-Frequency Regions during Atrial Fibrillation
}

\author{
Noninvasive Estimation of Fibrillation Frequencies
}

Pedrón-Torrecilla Jorge, Ph.D. ${ }^{1}$, Rodrigo Miguel, M.S. ${ }^{1}$, Climent Andreu M, Ph.D. ${ }^{2}$, Liberos Alejandro, M.S. ${ }^{1}$, Pérez-David Esther ${ }^{2}$, M.D. ${ }^{2}$, Bermejo Javier, M.D. ${ }^{2}$, Arenal Ángel, M.D ${ }^{2}$, Millet José, Ph.D. ${ }^{1}$, Fernández-Avilés Francisco, M.D., Ph.D. ${ }^{2}$, Berenfeld Omer, Ph.D., ${ }^{3}$, Atienza Felipe, M.D., Ph.D. ${ }^{2}$, Guillem María S, Ph.D. ${ }^{1}$

\author{
${ }^{1}$ ITACA, Universitat Politècnica de València, Valencia, Spain \\ ${ }^{2}$ Cardiology Department, Hospital General Universitario Gregorio Marañón, Instituto de \\ Investigación Sanitaria Gregorio Marañón, Madrid, Spain \\ ${ }^{3}$ Center for Arrhythmia Research, University of Michigan, Ann Arbor, U.S.A
}

\section{Address for correspondence:}

Maria S. Guillem, ITACA, Universidad Politécnica de Valencia, Camino de Vera sn, 46022 Valencia (Spain), tel.: +34963877968, email: mguisan@eln.upv.es or Felipe Atienza, Cardiology Department, Hospital General Universitario Gregorio Marañón, C/ Dr Esquerdo 46, 28007, Madrid (Spain), tel.: +34915868281, email: fatienzaf@secardiologia.es.

\section{Authors contributions:}

Mr. Pedron-Torrecilla, Dr. Climent, Dr. Guillem, Dr. Atienza: Concept/design. Mr. Pedron-Torrecilla, Mr. Liberos, Mr. Rodrigo: Data analysis/interpretation. Dr. Pérez-David, Dr. Bermejo, Dr. Atienza, Dr. Arenal, Dr. Climent: Data collection. Mr. Pedron-Torrecilla, Dr. Guillem, Dr. Climent: Drafting article. Mr. PedronTorrecilla, Dr. Climent, Dr. Guillem, Dr. Berenfeld, Dr. Atienza, Dr. Fernández-Avilés, Dr. Millet, Dr. PérezDavid, Dr. Bermejo: Critical revision and approval of article. Funding secured by Dr. Fernández-Avilés, Dr. Atienza and Dr. Millet. 


\section{Abstract}

Introduction -Ablation of high dominant frequency (DF) sources in patients with atrial fibrillation (AF) is an effective treatment option for paroxysmal AF. The aim of this study was to evaluate the accuracy of noninvasive estimation of DF and electrical patterns determination by solving the inverse problem of the electrocardiography.

Methods-Four representative AF patients with left-to-right and right-to-left atrial DF patterns were included in the study. For each patient, intracardiac electrograms from both atria were recorded simultaneously together with 67-lead body surface recordings. In addition to clinical recordings, realistic mathematical models of atria and torso anatomy with different DF patterns of AF were used. For both mathematical models and clinical recordings, inverse-computed electrograms were compared to intracardiac electrograms in terms of voltage, phase and frequency spectrum relative errors.

Results - Comparison between intracardiac and inverse computed electrograms for AF patients showed $8.8 \pm$ $4.4 \%$ errors for DF, $32 \pm 4 \%$ for voltage and $65 \pm 4 \%$ for phase determination. These results were corroborated by mathematical simulations showing that the inverse problem solution was able to reconstruct the frequency spectrum and the DF maps with relative errors of $5.5 \pm 4.1 \%$, whereas the reconstruction of the electrograms or the instantaneous phase presented larger relative errors (i.e. $38 \pm 15 \%$ and $48 \pm 14 \%$ respectively, $\mathrm{p}<0.01)$.

45 Conclusions - Non-invasive reconstruction of atrial frequency maps can be achieved by solving the inverse problem of electrocardiography with a higher accuracy than temporal distribution patterns.

Key words: atrial fibrillation, noninvasive mapping, inverse problem, body surface potential mapping, dominant frequency 


\section{Introduction}

The use of advanced signal analysis methods has shown that AF is maintained by high-frequency sources located at the junction of the atria with the left pulmonary veins (PV) or at others sites of the atria, both in animal models and in humans [1-7].

55 Several clinical studies have shown that, instead of empirically targeting the PVs [8] AF may be eliminated by directly ablating AF-driving sources or "rotors," that maintain the fibrillation with a hierarchical pattern from dominant frequency (DF) regions [9-11]. Thus, non-invasive identification of high frequency sources location prior to the ablation procedure could be used to select patients and to guide the ablation procedure.

Previous studies on inverse problem resolution during AF have shown paradoxically simple activation patterns [12-13] which do not correspond with the complex propagation patterns recorded either in animal models $[1,2,14]$ or epicardially in patients $[4,15]$ and have not been validated with simultaneously recorded potentials. For these reasons, the acceptance of the inverse-problem resolution as a guidance for AF ablation is still controversial. We have recently described the limitations encountered during the estimation of detailed voltage and phase propagation patterns during AF from noninvasive recordings [16]. In contrast, activation frequencies computed using spectral analysis has shown to be preserved on the torso surface [17]. The aim of the present study is to evaluate the performance of the inverse problem solution to detect and characterize intracardiac DF distributions during AF. To this purpose, we: 1) determined the feasibility and accuracy of computing dominant frequency (DF) maps from noninvasive recordings in AF patients in which surface and endocardial potentials were simultaneously acquired; and 2) assessed the accuracy of the inverse problem interpretation of the recorded body surface potentials in patients. 


\section{Methods}

\section{Simultaneous body surface and intracardiac recordings in AF patients}

Four patients admitted for ablation of drug-refractory paroxysmal AF ( males, $46.5 \pm 6.4$ years old) were studied. The ablation protocol as previously described was approved by the Institutional Ethics Committee of our institution and both patients gave informed consent [17]. Three patients arrived in sinus rhythm and AF was induced using electrical burst pacing [18].

80 In order to reconstruct the heart surface electrical activity by solving the inverse problem of the electrocardiography, multichannel electrocardiograms (ECGs) were recorded with a custom-made vest with 67 chest ECG leads by using the Body Surface Potential (BSPM) technique [17]. The geometry of the atria and torso of each patient was obtained by segmentation of Computed Axial Tomography (CAT) images. Specifically, images with a spatial resolution of $0.5 \mathrm{~mm}$ were acquired prior to the ablation procedure and segmented by using 3D Slicer [19].

Body surface recordings were simultaneously obtained with intracardiac EGMs. In two patients, intracardiac EGMs were obtained from the following catheters: (1) a standard tetrapolar catheter in the right atrial appendage (RAA); (2) a roving Navistar catheter (3.5-mm tip, 2-5-2 interelectrode distance; Thermo-Cool, Biosense-Webster, Diamond Bar, CA) used to obtain a DF map of both atria by sequentially obtaining EGMs at different atrial sites (>200 points) for at least 5 seconds and (3) a decapolar circular mapping Lasso catheter (Biosense-Webster, Diamond Bar, CA) placed either at the left superior pulmonary vein (LSPV) or right superior pulmonary vein (RSPV). Biatrial intracardiac signals were sequentially acquired at a sampling rate of $977 \mathrm{~Hz}$ and dominant frequency (DF) analysis was performed in real time using a CARTO navigation system with embebbed spectral capabilities (CARTO XP, version 7.7; Biosense-Webster, Diamond Bar, CA). After

95 mapping both atria and once the highest DF site was identified, the ablation catheter was placed at that site (i.e. LSPV for patient 1 and RAA for patient 2) and a central venous bolus of adenosine (12-18 mg) was administered to produce significant transient atrioventricular block avoiding ventricular activity [16, 17]. 
In two additional patients, body surface recordings were obtained simultaneously with a 64-pole basket catheter (Constellation, Boston Scientific, Natick, MA) located sequentially on the right and left atria. Additionally, a standard tetrapolar catheter was placed in the coronary sinus (CS) and a 20-poles catheter in the opposite atrium to the basket catheter. With the basket catheter located inside each atrium, a central bolus of adenosine (12-18 mg) was administered.

\section{Computational models of the atria and torso}

In order to support our observations in patients in which we have a limited number of simultaneous recordings with a more complete mapping data, we made use of mathematical models in which the electrical activity is known for the entire atrial surface. Realistic mathematical models of the atria and torso with different AF impulse propagation patterns were used to evaluate the performance of the inverse problem solution by comparing the generated EGMs to those computed by solving the inverse problem of the electrocardiography.

The electric potentials on the epicardium were calculated from a realistic computerized model of the atria. The active tissue of the atria consisted of 577264 nodes that represent a realistic human morphology [20]. The action potential of each node was simulated by using a modified version of Courtemanche’s mathematical model which includes ionic currents, pumps and exchangers [21] in which $\mathrm{I}_{\mathrm{K}, \mathrm{Ach}}$ current was introduced [6, 16].

Atrial fibrillation in the realistic model was induced in-silico by a S1-S2 stimulation protocol. Mathematical

115 computations were performed by using an adaptive time-step solver on a Graphical Processing Unit (NVIDIA Tesla C2075 6G) [22]. Transmembrane potentials were computed for a simulation time of 4 seconds after stabilization of the model and were resampled to $1 \mathrm{kHz}$.

Simulated electrograms (EGMs) were computed by using transmembrane potentials according to equation 1 at $1 \mathrm{~mm}$ distance from the epicardial surface [21]:

$$
E G M=\sum_{\vec{r}}\left(\frac{\vec{r}}{r^{3}}\right) \cdot \vec{\nabla} V_{m}
$$


where $\vec{r}$ is the distance vector between the measuring point and a point in the tissue domain, $\vec{\nabla}$ denotes the gradient operator, and $V_{m}$ is the transmembrane potential.

The computed potentials on the epicardium $U_{A}$ were used to compute the body surface potentials $U_{T}$ by applying the forward problem of the electrocardiography by using the Boundary Element Method, as 125 described in equation 2:

$U_{T}=M U_{A}$

where $M$ is the field transfer matrix between the atrial surface $A$ and the torso surface $T$. Those surfaces were discretized by plane triangles and the three vertex of each triangle. The transfer matrix was calculated by using the Boundary Element Method as described in equation 3 [23]:

$$
M=\left[D_{T T}-G_{T A} G_{A A}^{-1} D_{A T}\right]^{-1} \cdot\left[G_{T A} G_{A A}^{-1} D_{A A}-D_{T A}\right]
$$

where $D_{X Y}$ is the coefficient matrix that represents the contribution of the potential of a bounding surface $Y$ to a surface $X$ and $G_{X Y}$ is the coefficient matrix representing the contribution of the voltage gradient of a bounding surface $Y$ to a surface $X$. Assuming that $Y$ is a surface with $N_{Y}$ nodes and $X$ is a surface with $N_{X}$ nodes.

135 Simulated ECGs were used to estimate the inverse computed epicardial electrograms (icEGM) by solving the inverse problem of electrocardiography as described below. In order to evaluate the performance of the inverse problem under a realistic noisy situation, icEGM were calculated before and after the addition of white noise in the computed ECG signals (i.e. signal to noise ratio of 10dB).

\section{Noninvasive Characterization of Epicardial AF Activity}

140 We estimated icEGM from both patient recordings and body surface voltages computed from mathematical models, by solving the inverse problem of the electrocardiography. The inverse problem was solved by computing the inverse of the field transfer matrix atrial-torso matrix:

$$
U_{A}=M^{-1} U_{T}
$$


Since $M$ is ill-conditioned its inverse matrix cannot be computed in terms of classical linear algebra. We

145 solved the system by using zero-order Tikhonov's method in which the potentials on the surface of the atria $U_{A}$ were estimated from the potentials on the torso according to equation 5. [24, 23, 26].

$U_{A}(\lambda)=\left(M^{t} M+\lambda I^{t} I\right)^{-1} M^{t} U_{T}$

where $M$ is the field transfer matrix between the atria and the torso and can be calculated according to the equation $4, I$ is the identity matrix and $\lambda$ is the regularization parameter. In order to estimate the optimal regularization parameter $\lambda$, an automatized version of the graphical method of the L-curve was used [25].

The accuracy of the icEGM was compared for the (1) voltage, (2) phase and (3) frequency domains. Specifically: 1) instantaneous phase was computed by applying the Hilbert’s transform [16]; 2) power spectral density and DF were computed by using Welch's periodogram with a Hamming window of 2 seconds, 50\% overlap and a resolution of $0.25 \mathrm{~Hz}$; and 3) voltage was obtained from preprocessed bipolar intracardiac recordings [27].

Accuracy of reconstructed EGMs was quantified by measuring the relative error between normalized voltage, phase and spectrum of recorded or modelled EGMs and icEGM. In AF patients, icEGMs were compared with the simultaneously recorded endocardial EGM in three simultaneously recorded catheters; RAA, LSPV and RSPV. In mathematical models, electrograms from 5988 points of the realistic atria were compared. Statistical significance $(\mathrm{p}<0.05)$ was assessed using the $T$-test mean difference between the relative errors for the three domains (i.e. voltage, phase and frequency) at each node in the atrial model. The relative error for each node in the voltage and phase domain was computed as the mean relative error over the 5-seconds signal. For the frequency domain, the relative errorfor each node was computed as the mean relative error in each frequency in the range from 3 to $20 \mathrm{~Hz}$.

\section{Results}

\section{Noninvasive Identification of Atrial DFs during AF}


Performance of the inverse problem for the voltage, phase and frequency domains was assesed by comparing

170 simultaneous intracardiac electrograms with the estimation of the inverse problem solution in AF patients with different distribution patterns of activation frequency. In addition, inverse computed DF maps were compared with DF maps obtained either previously (first two patients) or simultaneously (last two patients) to the body surface recordings.

In Figure 1A and Figure 1C, intracardiac EGMs of patient 1 with a left-to-right dominant frequency pattern 175 with increasing activation rates from the RA, to RSPV and LSPV at $5.75 \mathrm{~Hz}, 6.75 \mathrm{~Hz}$ and $7 \mathrm{~Hz}$, respectively are shown. Comparing the intracardiac EGMs (i.e. blue) with the icEGMs (i.e. red), there was a significant error for both the voltage and the phase, whereas the morphology of the power spectrum and the measured DF values were similar. In fact, similar DF values were found on the RA, the LSPV and the RSPV after solving the inverse problem to those obtained in the simultaneous EGMs. CARTO DF maps and inverse-computed

180 DF maps also showed a good correspondence since the same hierarchical activation rate pattern (i.e. right to left frequency gradient) can be inferred from both maps. The actual activation frequencies, however, cannot be directly compared since they were not acquired simultaneously and the displayed surface maps correspond to the time of the adenosine infusion and adenosine is known to accelerate atrial frequencies, especially at the driving sites [6].

Patient 2 (Figure 1B and Figure 1D) presented a right-to-left dominant frequency pattern that was still present after isolation of PVs. with activation frequencies progressively decreasing from the RA, to RSPV and LSPV at $8 \mathrm{~Hz}, 6.25 \mathrm{~Hz}$ and $5.75 \mathrm{~Hz}$, respectively. Frequencies estimated from the icEGMs presented a similar activation pattern and frequency values as those measured invasively, whereas the reconstruction of the EGMs or the instantaneous phases presented a lower similitude. As shown in Figure 1F, these results were consistent with the CARTO maps, demonstrating a high similarity to the reconstructed DF maps, that also presented similar high dominant frequency distributions in the RA and LSPV and a right-to-left frequency gradient. Systematic comparison between intracardiac and icEGMs for the two AF patients corroborated those results with significantly lower relative error for frequency spectrum estimation than for the voltage or the instantaneous phase (i.e. $8.8 \pm 4.4 \%$ vs. $32 \pm 4 \%$ and $65 \pm 4 \%$ respectively, $\mathrm{p}<0.01$ ), as depicted in Figure $1 \mathrm{G}$. 
195 Additionally, the inverse problem allowed the reconstruction of the entire atrial DF maps for the same time interval as shown in Figure 1C and Figure 1D. Global DF map determination enabled the location of the highest DF site in the LSPV for patient 1 and in the RAA for patient 2, which is consistent with the DFs measured in the intracardiac recordings.

In two additional patients (patients 3 and 4), DF maps were further validated by comparing inverse-computed

200 EGMs with simultaneously recorded signals obtained at $71.8 \pm 8.9$ sites per atrium (total of 287 sites). Results of the comparison of inverse-computed and recorded EGMs are presented in Figure 2. Overall, DF maps allowed the identification of the highest DF site (in the right atrium of both patients) and the direction of the DF gradient, although the DF match at all sites was not perfect. Again, accuracy in the frequency domain, with a $12.8 \pm 3.2 \%$ error was higher than in the voltage and phase domains, with relative errors equal to 35.8 $205 \pm 6.6 \%$ and $65.4 \pm 4.1 \%$, respectively.

\section{Accuracy of the Inverse Problem Resolution}

In Figure 3, a mathematical model with a left-to-right DF gradient is shown. In this case, the DF gradient is governed by a rotor located at the left atrium as visible from the voltage and phase maps (Figure 3A). Under an ideal situation, in which the representation of the cardiac activity in the torso and the subsequent inverse problem resolution are performed without the addition of noise, inverse-computed voltages and phases represent a smoothened version of original epicardial potentials. However, while some activation wavefronts are properly estimated other activation wavefronts are missed (icEGMs in Figure 3B). On the other hand, dominant frequencies are accurately estimated by solving the inverse problem in most parts of the atria except for certain sites near the inter-atrial septum.

215 Epicardial potentials reconstructed after addition of noise at $10 \mathrm{~dB}$ SNR present an increased smoothening of both voltage distribution and phase to an extent at which activation wavefronts do not match after forward and inverse problem resolution (Figure 3C). Dominant frequency estimation was again performed accurately for most of the atrial surface. 
Figure 4 shows a mathematical model with a dominant rotor located at the right atrium giving rise to a right-

220 to-left DF gradient. As in the previous example, the resolution of the inverse problem produced a simplified version of the activation pattern of the potential and phase maps, especially under a realistic scenario with a signal to noise ratio of $10 \mathrm{~dB}$. Nevertheless, the addition of the noise did not modify the DF map.

The accuracy of the icEGM to represent the voltage, phase and frequency domains of original EGMs was systematically analyzed with and without the addition of white noise (Figure 5). As shown in Figure 5A, 225 error obtained for the DF distribution error was lower than the error obtained for the voltage and phase domains, both in the absence and presence of noise (i.e. $\mathrm{p}<0.01$ ). Notice that the addition of noise produced increased relative errors for the three domains. Nevertheless, the error increase was significantly smaller in the frequency domain than in the other two domains (i.e. $\mathrm{p}<0.01$ ), confirming our hypothesis that the frequency domain was the most robust parameter against noise.

230 The effect of noise in the performance of the inverse problem resolution can also be observed in Figure 5B, where a representative example of EGM from the model in Figure 3 is shown. In the absence of added noise, reconstructed EGMs matched modelled EGMs, although sharp voltage transitions were smoothened. However, after the addition of noise to surface potentials, a more pronounced filtering effect of higher order spectral components was observed, resulting in spatial smoothening and a poorer estimation of voltages and

235 phases. This effect might be due to the need to select a higher regularization parameter with added noise. Notice that smoothening produced a non-linear modification of the instantaneous phases for each instant which may explain the poor spatiotemporal reconstruction of the AF propagation patterns shown in Figure 3. In the spectral domain, the dominant peak was well preserved with a reduced power of higher order spectral components confirming that, although the inverse problem may have limitations to reconstruct AF propagation patterns, it allows an accurate reconstruction of DF maps. 


\section{Discussion}

The main finding of the present study is that noninvasive estimation of activation frequencies in the atria by solving the inverse problem of the electrocardiography is feasible and can be used to accurately compute activation frequency maps during AF. Here, we show that inverse quantification of atrial DF is more accurate than estimation of voltage distributions or their instantaneous phase.

\section{Non-invasive Estimation of Atrial Activation Patterns}

The inverse problem of the electrocardiography was first applied for solving the electrical activity in the atria

250 for the determination of earliest activation sites during atrial ectopies or pacing [13, 28, 29]. Solution of the inverse problem during non-fibrillatory rhythms has already been proven to be accurate by comparing inverse-computed and intracardially recorded isochronal maps. However, inverse-computed isopotential maps show paradoxically simple patterns during AF that have not been validated with simultaneous intracardiac data $[12,13]$. According to our results, voltage maps may not be inverse-reconstructed accurately, because

255 there is a trade-off between the smoothening introduced by large regularization parameters and an overreconstruction of noise in the computed electrograms due to small regularization parameters and, consequently, the optimal regularization parameters tended to underestimate the complexity of AF patterns. As we have previously shown [16], the simplified potentials that reach the torso surface may still contain the most relevant features of rotational drivers of AF, which may explain the reported success of the inverseguided ablation of rotors [12]. However, these results still need to be independently validated with simultaneous intracardiac mapping by other laboratories.

\section{Non-invasive Estimation of Atrial Dominant Frequencies}

There is experimental and clinical data supporting that in many cases AF is maintained by a region with the highest activation rate $[9,11,30]$. Ablation of the highest DF in the atria has shown to be an effective therapy for terminating with the arrhythmia [9]. We have recently demonstrated in a randomized clinical trial that in patients with paroxysmal AF, ablation of high frequency sites only is non-inferior and safer that empirically 
performing circumferential ablation of all PVs. Interestingly, this result was obtained isolating only a mean of 2.22 \pm 1.1 PVs [10].

270 Atrial dominant frequencies have been estimated from surface recordings for decades [31] and have shown good correlation with global intracardiac atrial DFs [32, 33]. In a more recent study, we have shown that extensive recordings of surface potentials by BSPM allow detecting the highest dominant frequencies and not only the global activation rate of the overall atrial tissue [17]. However, surface frequency maps only provide a rough estimation of the location of the highest DF site.

275 Here we have shown that solution of the inverse problem of the electrocardiography allows locating this highest DF site in a patient-specific model of the atria, observation that has been validated by using simultaneously recorded intracardiac electrograms. Our simulation study suggests that inverse estimation of the atrial activation frequency of atrial electrograms during AF based on the dominant frequency calculation is more accurate than the estimation of temporal-based features such as isopotential or phase maps, especially under a realistic noise scenario. The smoothing effect of the torso at non negligible distances from the atria results in the summation of nearby potentials [16]. The smoothing effect during classic inverse problem solution using quadratic regularization parameters is required in the presence of noise, but introduces phase distortions that preclude an accurate reconstruction of spatiotemporal variations of non-stochastic phenomena like AF. Nevertheless, the present study demonstrates that the inverse problem can be used to accurately reconstruct the DF map and consequently detect the region of the atria that hierarchically maintains AF.

\section{Limitations of the study}

In this study we have validated the estimation of inverse-computed DF maps with a limited sample of patients. However, these patients represent different locations of the highest DF site location (i.e. right vs. left) and may be representative of a broader range of atrial DF patterns. In some these patients we do not have simultaneous information of the entire atrial surface and, in order to overcome this limitation, we used mathematical models in which the activity in the entire atria is known to support our observations regarding the superiority of DF estimation over time-based metrics. Although the mathematical models employed may be over-simplistic, they were complex enough to validate our hypothesis: that the main spectral components 
(namely, the DF) are better preserved than the morphology of the EGMs or their phases. Validation with more complex mathematical models would only make these results more significant.

Finally, analyzed ECG segments correspond to an intravenous adenosine injection which does not only produce a transient atrio-ventricular block but also accentuates the highest frequencies, without affecting the atrial frequency gradient or the location of the highest DF site [6, 34]. For this reason, the estimated frequencies may be higher than those that would be recorded in basal conditions.

300

\section{Conclusion and Clinical Implications}

Atrial high frequency sources can be identified noninvasively by solving the inverse problem of electrocardiography with a higher accuracy than the morphology of potentials or their phase. Noninvasive computation of DF maps prior to and during an ablation procedure may help in patient selection and personalized procedure planning.

\section{Acknowledgements}

Supported in part by: Spanish Ministry of Education (FPU-2012 and FPU-2010); Instituto de Salud Carlos III (Ministry of Economy and Competitiveness, Spain: PI12/00993-00407; PI13-01882, PI13-00903 and

PI14/00857); Spanish Society of Cardiology (Grant for Clinical Research in Cardiology 2015); the Universitat Politècnica de València through its research initiative program; the Generalitat Valenciana (PROMETEO 2010/093 and ACIF/2013/021); the Ministerio de Economia y Competitividad, Red RIC; the Coulter Foundation from the Biomedical Engineering Department (University of Michigan); the Gelman Award from the Cardiovascular Division (University of Michigan); the National Heart, Lung, and Blood Institute grants (P01-HL039707, P01-HL087226 and R01-HL118304), and the Leducq Foundation. 


\section{References}

1. Mandapati R, Skanes A, Chen J, Berenfeld O, Jalife J: Stable microreentrant sources as a mechanism of atrial fibrillation in the isolated sheep heart. Circulation 2000; 101:194-199.

2. Mansour M, Mandapati R, Berenfeld O, Chen J, Samie FH, Jalife J: Left-to-right gradient of atrial frequencies during acute atrial fibrillation in the isolated sheep heart. Circulation 2001; 103:2631-2636.

3. Sanders P, Berenfeld O, Hocini M, Jaïs P, Vaidyanathan R, Hsu LF, Garrigue S, Takahashi Y, Rotter M, Sacher F, Scavée C, Ploutz-Snyder R, Jalife J, Haïssaguerre M: Spectral analysis identifies sites of high-frequency activity maintaining atrial fibrillation in humans. Circulation 2005; 112:789-797.

4. Sahadevan J, Ryu K, Peltz L, Khrestian CM, Stewart RW, Markowitz AH, Waldo AL: Epicardial mapping of chronic atrial fibrillation in patients: preliminary observations. Circulation 2004; 110:32933299.

5. Lazar S, Dixit S, Marchlinski FE, Callans DJ, Gerstenfeld EP: Presence of left-to-right atrial frequency gradient in paroxysmal but not persistent atrial fibrillation in humans. Circulation 2004; 110:3181-3186.

6. Atienza F, Almendral J, Moreno J, Vaidyanathan R, Talkachou A, Kalifa J, Arenal A, Villacastín JP, Torrecilla EG, Sánchez A, Ploutz-Snyder R, Jalife J, Berenfeld O: Activation of inward rectifier potassium channels accelerates atrial fibrillation in humans-Evidence for a Reentrant mechanism. Circulation 2006; 114:2434-2442.

7. Lin WS, Tai CT, Hsieh MH, Tsai CF, Lin YK, Tsao HM, Huang JL, Yu WC, Yang SP, Ding YA, Chang MS, Chen SA: Catheter Ablation of Paroxysmal Atrial Fibrillation Initiated by Non-Pulmonary Vein Ectopy. Circulation 2003; 107:3176-3183.

8. Calkins H, Brugada J, Packer DL, Cappato R, Chen SA, Crijns HJ, Damiano RJ Jr, Davies DW, Haines DE, Haissaguerre M, Iesaka Y, Jackman W, Jais P, Kottkamp H, Kuck KH, Lindsay BD, Marchlinski FE, McCarthy PM, Mont JL, Morady F, Nademanee K, Natale A, Pappone C, Prystowsky E, Raviele A, Ruskin JN, Shemin RJ: HRS/EHRA/ECAS Expert consensus statement on catheter and surgical 
ablation of atrial fibrillation: Recommendations for personnel, policy, procedures and follow-up. Heart Rhythm 2007; 4:816-861.

9. Atienza F, Almendral J, Jalife J, Zlochiver S, Ploutz-Snyder R, Torrecilla E, Arenal A, Kalifa J, 345 Fernandez Aviles F, Berenfeld O: Real-time dominant frequency mapping and ablation of dominant frequency sites in atrial fibrillation with left-to-right frequency gradients predicts long-term maintenance of sinus rhythm. Heart Rhythm 2009; 6:33-40.

10. Atienza F, Almendral J, Ormaetxe JM, Moya A, Martínez-Alday JD, Hernández-Madrid A, Castellanos E, Arribas F, Arias MÁ, Tercedor L, Peinado R, Arcocha MF, Ortiz M, Martínez-Alzamora N, Arenal A, Fernández-Avilés F, Jalife J: Comparison of Radiofrequency Catheter Ablation of Drivers versus Circumferential Pulmonary Vein Isolation in Patients with Atrial Fibrillation: A Noninferiority Randomized Multicenter RADAR-AF Trial. J Am Coll Cardiol 2014; 64:2455-2467.

11. Narayan SM, Krummen DE, Shivkumar K, Clopton P, Rappel WJ, Miller JM: Treatment of Atrial Fibrillation by the Ablation of Localized Sources. JACC 2012; 60:628-636.

355 12. Haissaguerre M1, Hocini M1, Denis A1, Shah AJ1, Komatsu Y1, Yamashita S1, Daly M1, Amraoui S1, Zellerhoff S1, Picat MQ1, Quotb A1, Jesel L1, Lim H1, Ploux S1, Bordachar P1, Attuel G1, Meillet V1, Ritter P1, Derval N1, Sacher F1, Bernus O1, Cochet H1, Jais P1, Dubois R1. Driver domains in persistent atrial fibrillation. Circulation. 2014 Aug 12;130(7):530-8.

13. Cuculich PS, Wang Y, Lindsay BD, Faddis MN, Schuessler RB, Damiano Jr RJ, Li L, Rudy Y: Noninvasive Characterization of Epicardial Activation in Humans With Diverse Atrial Fibrillation Patterns. Circulation 2010; 122:1364-1372.

14. Berenfeld O, Mandapati R, Dixit S, Skanes AC, Chen J, Mansour M, Jalife J: Spatially distributed dominant excitation frequencies reveal hidden organization in atrial fibrillation in the Langendorffperfused sheep heart. J Cardiovasc Electrophysiol 2000; 11:869-879. 

Electropathological substrate of long-standing persistent atrial fibrillation in patients with structural heart disease: longitudinal dissociation. Circ Arrhythm Electrophysiol 2010; 3:606-615.

16. Rodrigo M, Guillem MS, Climent AM, Pedrón-Torrecilla J, Liberos A, Millet J, Fernández-Avilés F, Atienza F, Berenfeld O: Body surface localization of left and right atrial high-frequency rotors in atrial fibrillation patients: a clinical-computational study. Heart Rhythm 2014; 11:1584-1591.

17. Guillem MS, Climent AM, Millet J, Arenal Á, Fernández-Avilés F, Jalife J, Atienza F, Berenfeld O: Noninvasive localization of maximal frequency sites of atrial fibrillation by body surface potential mapping. Circ Arrhythm Electrophysiol 2013; 6:294-301.

18. Atienza F, Calvo D, Almendral J, Zlochiver S, Grzeda KR, Martínez-Alzamora N, González-Torrecilla E, Arenal A, Fernández-Avilés F, Berenfeld O: Mechanisms of fractionated electrograms formation in the posterior left atrium during paroxysmal atrial fibrillation in humans. J Am Coll Cardiol 2011; 57:10811092.

19. Fedorov A, Beichel R, Kalpathy-Cramer J, Finet J, Fillion-Robin JC, Pujol S, Bauer C, Jennings D, Fennessy F, Sonka M, Buatti J, Aylward S, Miller JV, Pieper S,Kikinis R: 3D Slicer as an Image Computing Platform for the Quantitative Imaging Network. Magn Reson Imaging 2012; 30:1323-1341.

20. Harrild DM, Henriquez CS: A computer model of normal conduction in the human atria. Circ Res 2000; 87:E25-E36.

21. Courtemanche M, Ramirez RJ, Nattel S: Ionic mechanisms underlying human atrial action potential properties: Insights from a mathematical model. Am J Physiol -Heart Circul Physiol 1998; 275:H301H321.

22. Garcia-Molla V, Liberos A, Vidal A, Guillem MS, Millet J, Gonzalez A, Martinez-Zaldivar FJ, Climent AM: Adaptive step ODE algorithms for the 3D simulation of electric heart activity with graphics processing units. Comput Biol Med 2014; 44:15-26. 
23. Horácek BM, Clements JC: The Inverse Problem of Electrocardiography: A Solution in Terms of Singleand Double-Layer Sources on the Epicardial Surface. Math Biosci 1997; 144:119-154.

24. Dunavant DA: High degree efficient symmetrical Gaussian quadrature rules for the triangle. Int J Numer Meth Eng 1985; 21:1129-1248.

25. Hansen PC, O'Leary DP: The use of the L-curve in the regularization of discrete ill-posed problems. SIAM J Sci Stat Comput 1993; 14:1487-1503.

26. Tikhonov AN: On the solution of incorrectly posed problems and the method of regularization. Sov Math Dokl 1963; 4:1035-1038.

27. Botteron GW, Smith JM: Quantitative assessment of the spatial organization of atrial fibrillation in the intact human heart. Circulation 1996; 93:513-518.

28. Roten L, Pedersen M, Pascale P, Shah A, Eliautou S, Scherr D, Sacher F, Haïssaguerre M: Noninvasive Electrocardiographic Mapping for Prediction of Tachycardia Mechanism and Origin of Atrial Tachycardia Following Bilateral Pulmonary Transplantation. J Cardiovasc Electrophysiol 2012; 23:553555.

29. Pedrón-Torrecilla J, Climent A, Liberos A, Pérez-David E, Millet J, Atienza F, Guillem MS: NonInvasive Estimation of the Activation Sequence in the Atria during Sinus Rhythm and Atrial Tachyarrhythmia. Computing in Cardiology 2012; 39:901-904.

30. Lazar S, Dixit S, Callans DJ, Lin D, Marchlinski FE, Gerstenfeld EP: Effect of pulmonary vein isolation on the left-to-right atrial dominant frequency gradient in human atrial fibrillation. Heart Rhythm 2006; 3:889-895.

31. Bollmann A, Kanuru NK, McTeague KK, Walter PF, DeLurgio DB, Langberg JJ: Frequency analysis of human atrial fibrillation using the surface electrocardiogram and its response to ibutilide. Am J Cardiol 1998; 81:1439-1445. 
32. Dibs SR, Ng J, Arora R, Passman RS, Kadish AH, Goldberger JJ: Spatiotemporal characterization of atrial activation in persistent human atrial fibrillation: multisite electrogram analysis and surface electrocardiographic correlations: a pilot study. Heart Rhythm 2008; 5:686-693.

415

33. Petrutiu S, Sahakian AV, Fisher W, Swiryn S: Manifestation of left atrial events and interatrial frequency gradients in the surface electrocardiogram during atrial fibrillation: contributions from posterior leads. J Cardiovasc Electrophysiol 2009; 20:1231-1236.

34. Atienza F, Jalife J: Reentry and atrial fibrillation. Heart Rhythm 2007; 4:S13-16. 
Figure 1: Validation of the inverse problem during human AF for the voltage, phase and frequency domains. (A and B) Intracardiac (blue) and inverse-computed (red) EGMs at the right atrial appendage (RAA), Right Superior Pulmonary Vein (RSPV) and Left Superior Pulmonary Vein (LSPV) and their corresponding instantaneous phase and spectra for patient 1 (A), with a left-to-right DF gradient, and for patient 2 (B), with a right-to-left gradient. (C and D) Inverse-computed DF maps, posterior (right) and right lateral (left) biatrial views of patients 1 and 2, respectively. (E and F) CARTO maps of patients 1 and 2. (G) Numerical analysis of the relative error in the voltage, phase and frequency reconstruction in patients for the three selected recorded and inverse-computed EGMs for each patient.

Figure 2: Validation of inverse computed DF maps. Inverse computed $(A, C)$ and simultaneously recorded DF maps (B,D) for patients 3 and 4 in which a multipolar catheter was sequentially placed in the right and left atria. (E) Numerical analysis of the relative error in the voltage, phase and frequency reconstruction in both patients for a total of 287 EGMs recorded in both atria of the two patients $(71.8 \pm 8.9$ simultaneously recorded sites).

Figure 3: Inverse-computed voltage, phase and dominant frequency (DF) maps for simulated AF with a left-to-right DF gradient. (A) Voltage, phase and DF maps for generated epicardial electrograms (EGM). (B) Voltage, phase and DF maps for inverse computed electrograms (icEGM) without added noise. (C) Voltage, phase and DF maps for icEGM with added noise on surface potentials at $10 \mathrm{dBs}$ SNR.

Figure 4: Inverse-computed voltage, phase and dominant frequency (DF) maps for simulated AF with a right-to-left DF gradient. (A) Voltage, phase and DF maps for generated epicardial electrograms (EGM). (B) Voltage, phase and DF maps for inverse computed electrograms (icEGM) without added noise. (C) Voltage, phase and DF maps for icEGM with added noise on surface potentials at $10 \mathrm{dBs}$ SNR. 
Figure 5. Comparison between simulated and inverse-computed EGMs. (A) Relative error between inverse computed electrograms (icEGM) and original electrograms (EGM) with and without the addition of white noise to the surface electrocardiograms (i.e. signal to noise ratio (SNR) of 10dB). (B) Comparison between the original EGMs and icEGMs at a selected atrial site. 


\section{FIGURE 1}

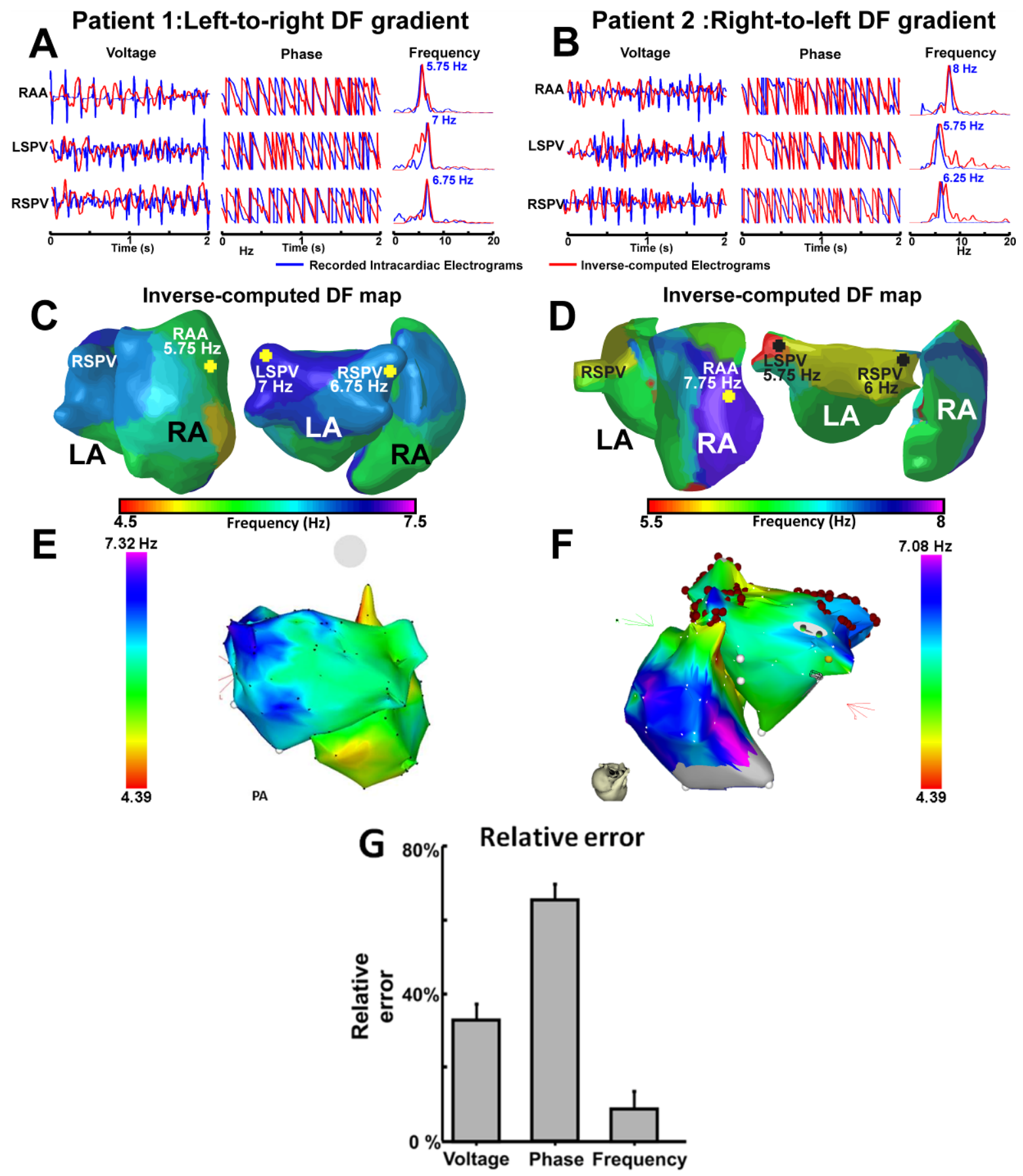


FIGURE 2
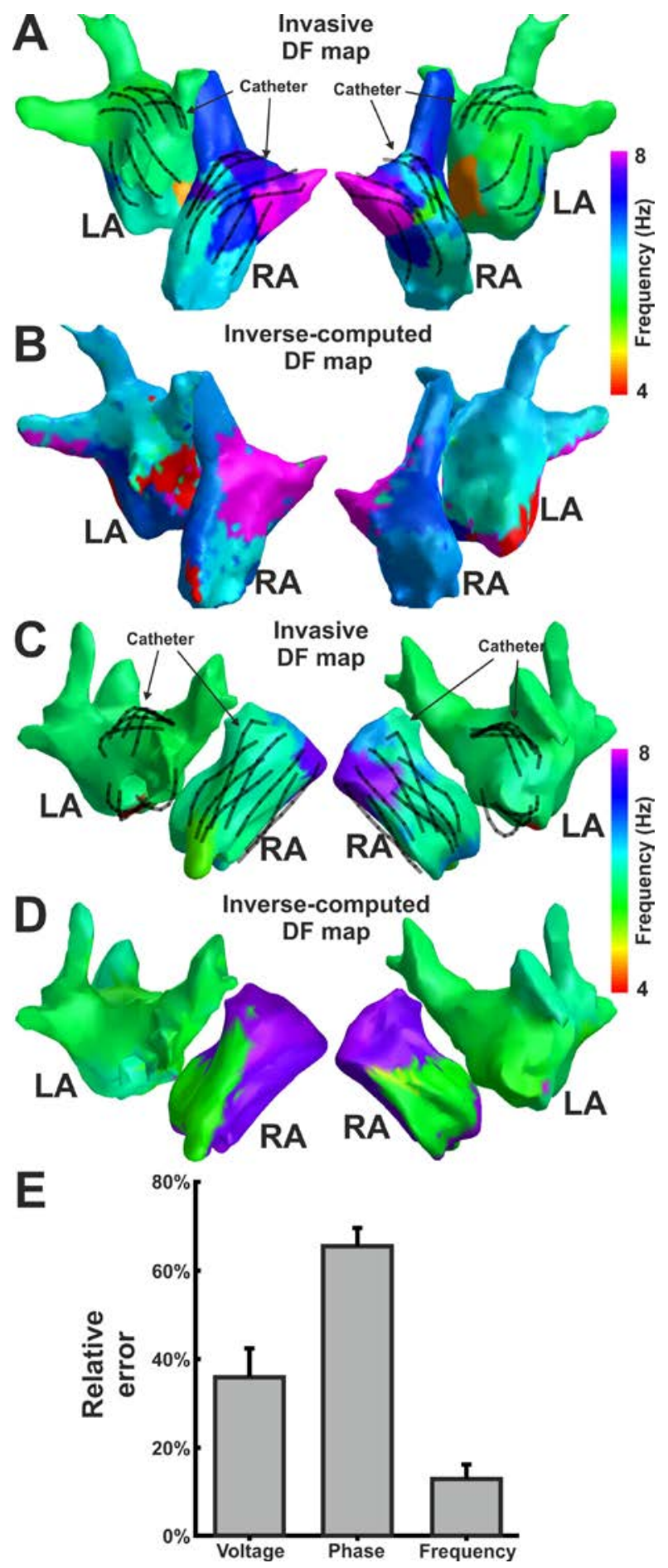


\section{FIGURE 3}

\section{Left-to-Right DF gradient}
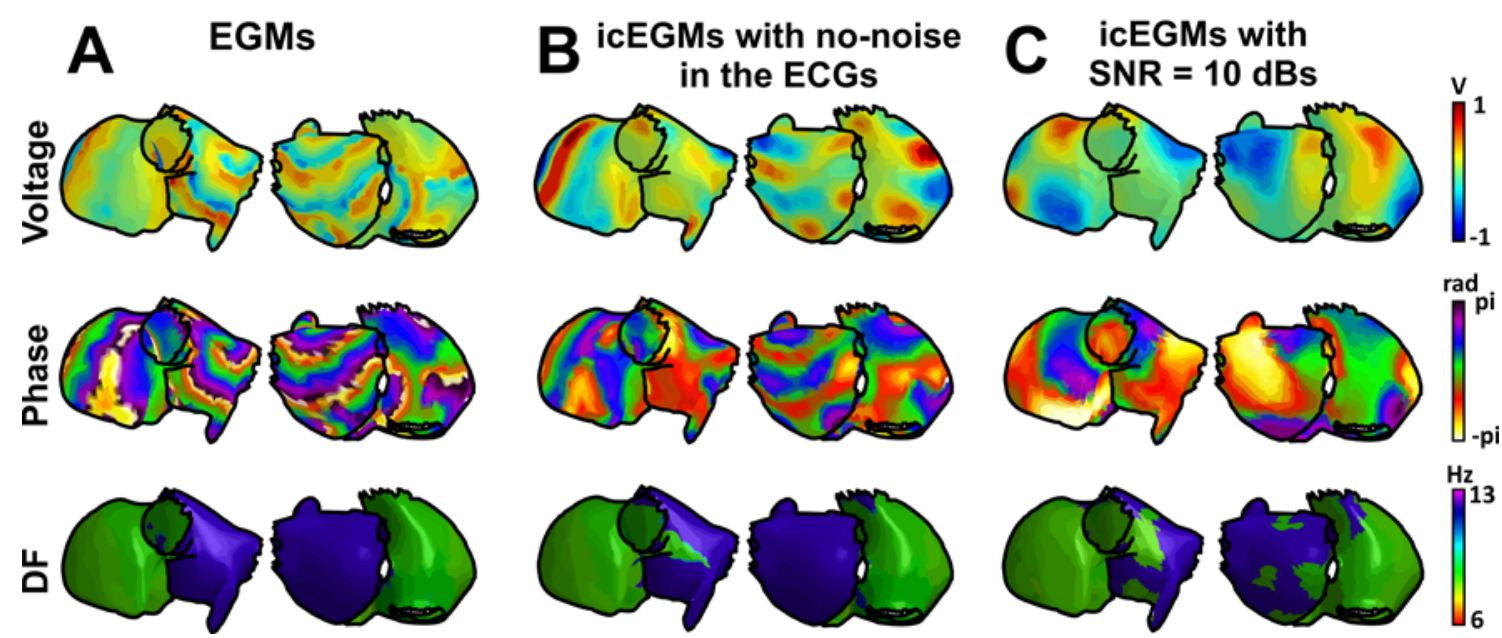


\section{Right-to-Left DF gradient}

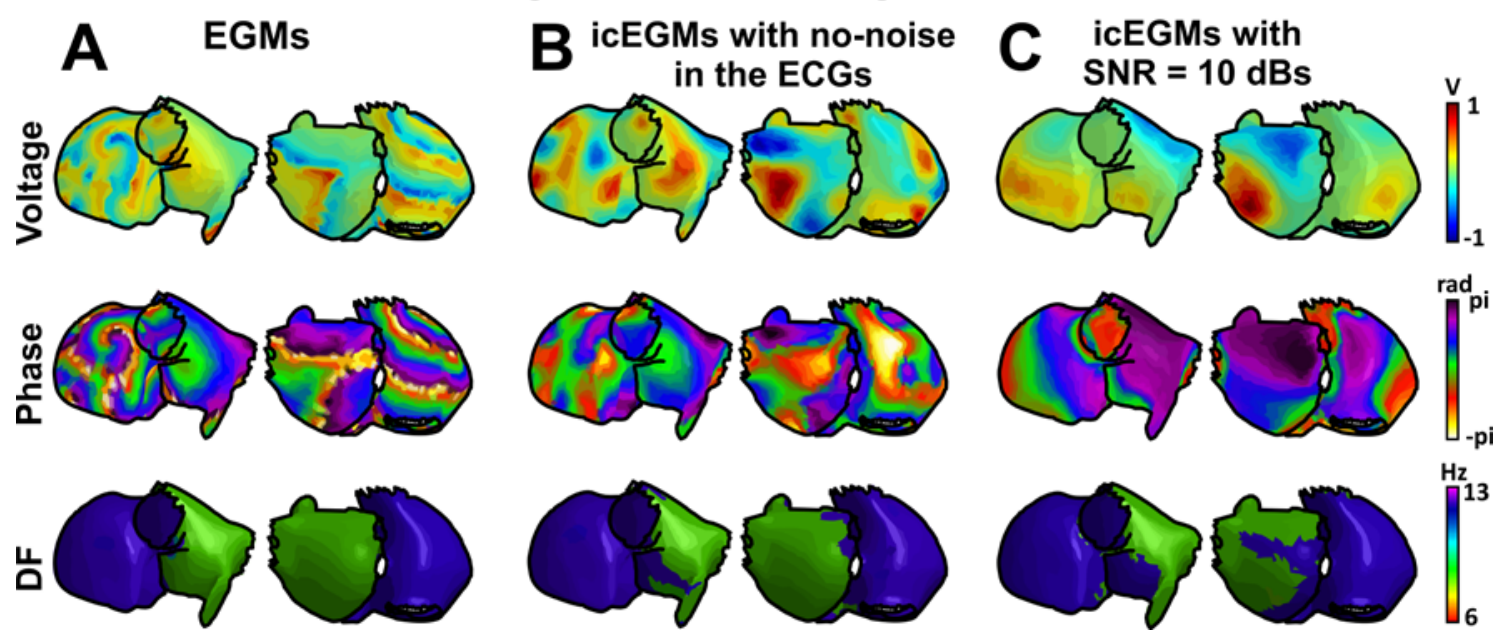




\section{FIGURE 5}
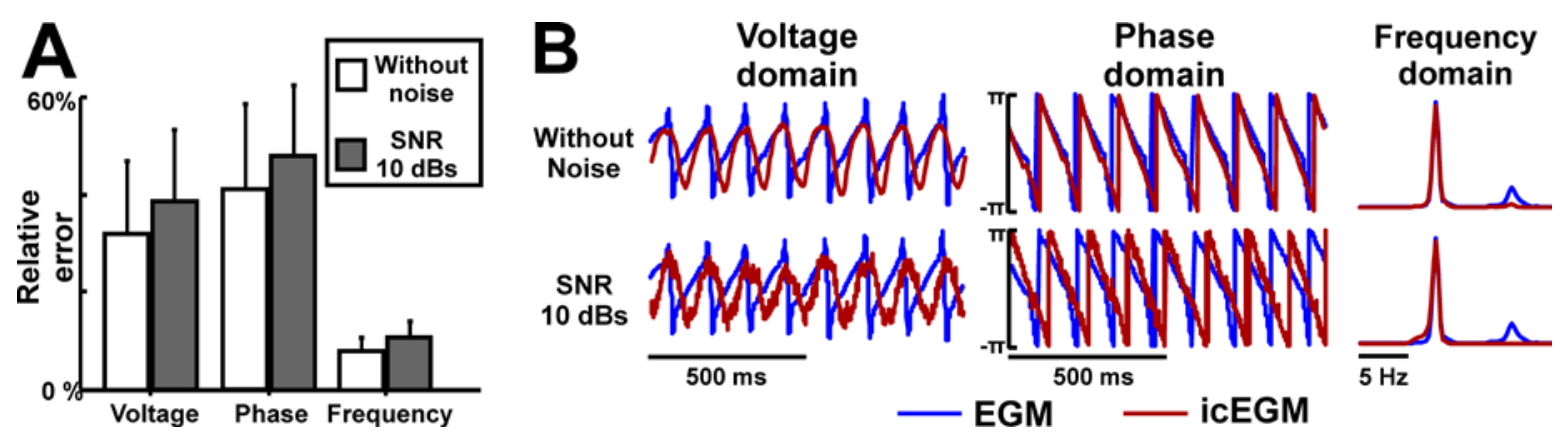